\title{
Giant magnetic anisotropy at the nanoscale: Overcoming the superparamagnetic limit
}

\author{
A. Hernando, ${ }^{1, *}$ P. Crespo, ${ }^{1}$ M. A. García, ${ }^{1}$ E. Fernández Pinel, ${ }^{1}$ J. de la Venta, ${ }^{1}$ A. Fernández, ${ }^{2}$ and S. Penadés ${ }^{3}$ \\ ${ }^{1}$ RENFE-UCM-CSIC and Departamento de Física de Materiales UCM Instituto de Magnetismo Aplicado, P.O. Box 155, Las Rozas, \\ 28230 Madrid, Spain \\ ${ }^{2}$ Instituto de Ciencia de Materiales de Sevilla CSIC-UNSE, Américo Vespucio s/n, 41092 Sevilla, Spain \\ and Departamento de Quimica Inorgánica, Universidad de Sevilla, Spain \\ ${ }^{3}$ Grupo Carbohidratos, Laboratorio of Glyconanotechnology IIQ-CSIC, Américo Vespucio s/n, 41092 Sevilla, Spain
}

(Received 7 May 2006; revised manuscript received 22 June 2006; published 17 August 2006)

It has been observed for palladium and gold nanoparticles that the magnetic moment at a constant applied field does not change with temperature over the range comprised between 5 and $300 \mathrm{~K}$. These samples, with sizes smaller than $2.5 \mathrm{~nm}$, exhibit remanent magnetization up to room temperature. The existence of permanent magnetism up to so high temperatures has been explained as due to the blocking of a local magnetic moment by giant magnetic anisotropies. In this Brief Report we show, by analyzing the anisotropy of thiol capped gold films, that the orbital momentum induced at the surface conduction electrons is crucial to understand the observed giant anisotropy. The orbital motion is driven by a localized charge and/or spin through spin-orbit interaction, which reaches extremely high values at the surfaces. The induced orbital moment gives rise to an effective field of the order of $10^{3} \mathrm{~T}$ that is responsible for the giant anisotropy.

DOI: 10.1103/PhysRevB.74.052403

PACS number(s): 75.30.Gw, 75.75.+a, 75.10.-b

Magnetism at nanoscale presents surprising experimental results. Gambardella et al. found that single Co atoms deposited onto Pt surfaces show a remarkable magnetic anisotropy with an easy axis perpendicular to the surface. ${ }^{1}$ The corresponding anisotropy constant was estimated to be close to $10 \mathrm{meV}$ per $\mathrm{Co}$ atom, larger than that corresponding to the harder magnetic material ( $2 \mathrm{meV}$ per $\mathrm{Co}$ atom for $\mathrm{SmCo}_{5}$ ). For $1.4 \mathrm{~nm}$ thiol capped gold nanoparticles (NPs) a permanent magnetism was detected up to room temperature ${ }^{2,3}$ whereas a similar behavior had been previously reported for $2.4 \mathrm{~nm}$ palladium NPs. ${ }^{4,5}$ For the case of gold the appearance of magnetism was completely amazing provided the diamagnetic character of bulk samples and the low value of its density of states at the Fermi level. However, more unexpected was that NPs with sizes smaller than $2.4 \mathrm{~nm}$ could exhibit blocked magnetism at $300 \mathrm{~K}^{2-5}$ Moreover, the thermal dependence of magnetization for Pd and Au NPs is very weak between 5 and $300 \mathrm{~K}$. By assuming a first order kinetics for the relaxation of the magnetic moments and an attempted frequency factor equal to approximately $10^{10} \mathrm{~s}^{-1}$, the anisotropy constant for a particle $2 \mathrm{~nm}$ size with blocking temperature above $300 \mathrm{~K}$ should be at least of $10^{9} \mathrm{~J} \mathrm{~m}^{-3}$ that corresponds to approximately $0.4 \mathrm{eV}$ per atom. That is indeed an enormous value compared not only to the normal values for the harder magnetic materials but also to the value reported in Ref. 1.

Recently, Carmeli et al. ${ }^{6,7}$ found that thiol capped gold surfaces exhibit a giant paramagnetism with $50 \mu_{B}$ (Bohr magnetons) per atom and an easy axis also perpendicular to the surface, i.e., along the $z$ axis. In order to analyze the giant values of the anisotropy one can better use the results obtained for thiol capped gold films where the easy axis is uniform over the whole surface and the local anisotropy merges macroscopically.

We have prepared Au films capped with Lewis conjugate onto glass substrates by a template-stripped gold method with atomically flat surfaces. ${ }^{8}$ Lewis conjugate, that links via a covalent bond to a Au surface through sulphur similarly to thiols, is known to enhance self assembly effects. ${ }^{9}$ Initially, the magnetic properties of the bare substrate were measured. As Fig. 1(a) shows, the substrates exhibit a paramagnetic behavior at low temperature that turns to a diamagnetic one over $150 \mathrm{~K}$. The resulting magnetization curves were identical when measured with the magnetic field applied either in plane or perpendicular to the surface. This is the typical behavior arising from magnetic impurities whose magnetization decreases with temperature. Figure 1(b) shows the magnetization curves measured at $5 \mathrm{~K}$ before and after chemisorption of the Lewis conjugate. When the field is applied in plane, the magnetization is the same as that before chemisorption, however applying the magnetic field perpendicular to the surface, the resulting magnetization curve was clearly different. Chemisorption induces a new contribution to the magnetization which can be obtained by subtracting the curves before and after the chemisorption process. Repeating the procedure at different temperatures, we found that the new contribution to the magnetization perpendicular to the surface induced by the chemisorption results, independent of temperature [Fig. 1(c)], in the range 5-300 K. For in-plane field there is no difference between the substrate and the sample with the Lewis conjugate chemisorbed in this range of temperature [Fig. 1(d)].

From this curves with the magnetic field perpendicular to the surface and considering that for the $\mathrm{Au}[111]$ surface there are $2 \times 10^{14}$ atoms $/ \mathrm{cm}^{2},{ }^{6}$ the magnetic moment per surface atom is about $100 \mu_{B}$ per atom. Two questions arise from these results: (a) the origin of this anisotropy and (b) the magnetization process provided that the coercivity of the magnetization curve along the easy axis is negligible as compared to the expected values of the anisotropy field.

Let us first show that the origin of the anisotropy is the same as the origin of the giant moments. In a recent paper ${ }^{10}$ the authors have explained this enormous magnetic moment as due to the orbital motion of the surface quasi-free electrons around the island of radius $\xi$ formed by self-assembled 

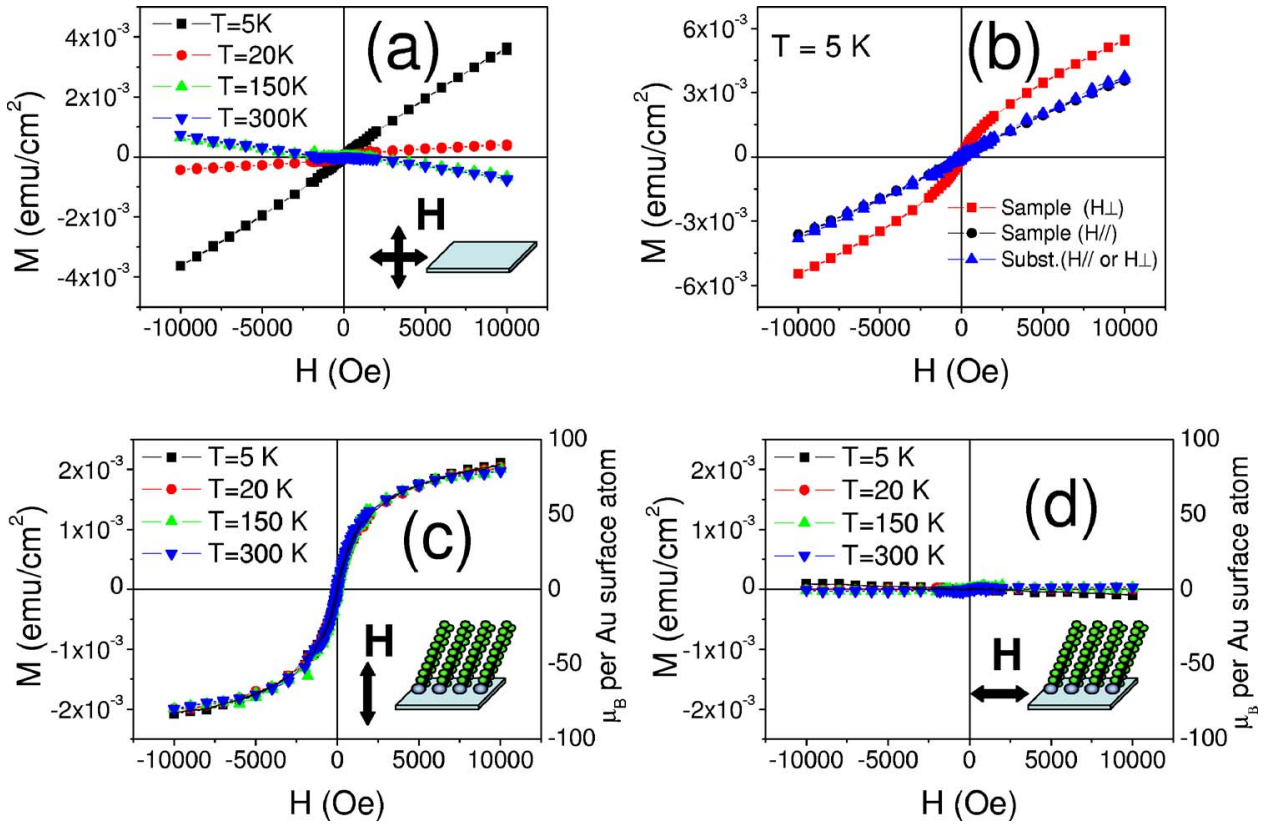

FIG. 1. (Color online) Magnetization curves for (a) the bare substrate with $H$ perpendicular to the surface; the same result was found for $H$ parallel; (b) the bare substrate and the sample with $H$ parallel and perpendicular at $5 \mathrm{~K}$. (c) The difference between the magnetization curves for the substrate and the sample with $H$ perpendicular to the surface and (d) $H$ parallel to the surface. thiols bonding. The presence of localized charge or localized spin drives the induction of orbital momentum at the conduction electrons to minimize the spin-orbit interaction, $\alpha_{r} \hbar^{2}$. The quantum number $l_{z}$ that minimizes the kinetics and spinorbit energy terms is easily obtained to be $l_{z}^{*}=m \xi^{2} \alpha_{r} \mathrm{~s}_{z}$ ( $m$ and $s_{z}$ hold for the mass and the third component of the spin of the free electron, respectively). The threshold strength of $\xi^{2} \alpha_{r}$, in order to trap an electron in the orbit, is that corresponding to $l_{z}^{*}=1$, i.e., the capture condition becomes $\xi^{2} \alpha_{r}>2 \times 10^{30} \mathrm{~kg}^{-1}{ }^{11}$ The strong spin-orbit interaction $\left(\alpha_{r} \hbar^{2}=0.4 \mathrm{eV}\right)$ at the gold surface ${ }^{12,13}$ together with the large radius of the ordered islands ${ }^{14}$ (more than $50 \mathrm{~nm}$ ) drive the induction of giant orbital momenta. Furthermore, if instead of local electric charge the surface holds a localized spin, with $z$ component $S_{z}$, the conduction electrons will also rotate around it by the effect of the spin-orbit coupling. Thus, if the island consists of a localized ordered arrangement of both spin and charge, the orbital momentum that minimizes the energy should be given by $l_{z}^{*}=m \xi^{2} \alpha_{r}\left(S_{z}+s_{z}\right)$, where we assume $\alpha_{r}$ to be the experimental spin-orbit coupling strength measured for the particular surface. The spin-orbit interaction per atom that couples the localized charge and spin to the spin and orbital momenta of the conduction electron can then be written as

$$
H_{\mathrm{s}-\mathrm{o}}=\alpha_{r} \hbar^{2} l_{z}\left(s_{z}+S_{z}\right),
$$

where $l_{z}$ and $s_{z}$ are, respectively, the angular and spin momenta of the conduction electrons per atom comprised within the orbit. ${ }^{10}$ The contribution to the surface magnetic moments comes from three sources (see Fig. 2): (a) the localized magnetic moment $g \mu_{B} J=g \mu_{B}(L+S)$, where $g$ is the Landé factor and $J$ is the total angular momentum of a localized electron (hereinafter for the sake of clarity we will consider $L=0$ ), (b) $\mu_{B} l_{z}$ is the orbital magnetic moment per atom induced on conduction electrons, and (c) $2 \mu_{B} s_{z}$ is the magnetic moment per atom associated with the spin of the conduction electrons with orbital momentum $l_{z}$. The total mo- mentum depends on the sign of $\alpha_{r}$, but for the case of giant orbital moments the spin contribution is negligible. The rest of the conduction electrons and those localized around the atomic cores give rise to the well determined diamagnetic susceptibility of gold. Note that relation (1) points out the existence of an effective exchange coupling between the orbital moment and the spins of both orbital trapped and localized electrons.

In order to confirm that the huge magnetic moments come from the orbital motion of conduction electrons and are not due exclusively to organic molecules, we prepared thiolcapped Si surfaces. For Si, both spin-orbit coupling and the number of conduction electrons are largely reduced with respect to Au. The magnetization curves corresponding to this sample, presented in Fig. 3, show a very weak magnetic signal (actually about the resolution of the equipment) indicating that, if exists, the magnetic moment is below $1 \mu_{B}$ per surface atom. Moreover, in a recent experiment, Carmeli et

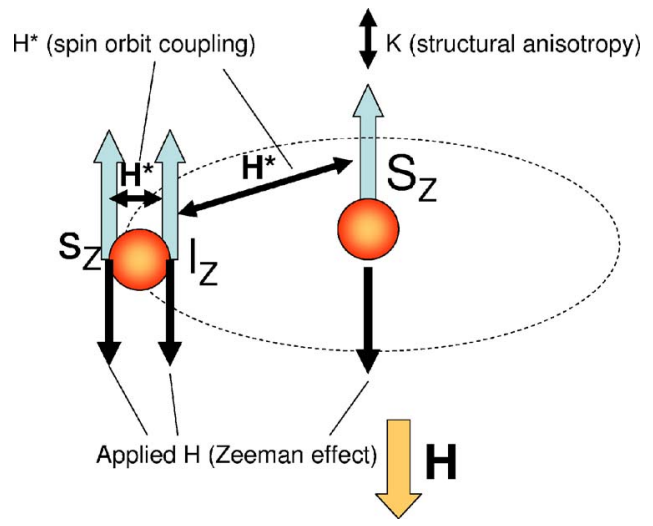

FIG. 2. (Color online) Scheme of the different magnetic moments and the interaction controlling their orientation. The relative orientation of $S_{Z}, s_{z}$, and $l_{z}$ is fixed by $H^{*}$. The structural anisotropy acts only on $S_{z}$, while the reversal magnetic applied field acts on all of them. 


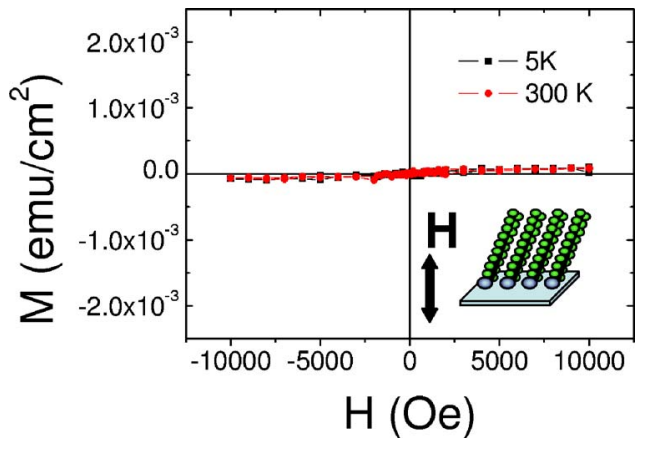

FIG. 3. (Color online) Magnetization curves for a thiol-capped Si substrate with the $H$ field applied perpendicular to the surface.

al. ${ }^{16}$ found giant magnetic moments on samples consisting of polyalanine molecules chemisorbed on Au surfaces but no giant moments were found when depositing those molecules onto $\mathrm{Si}$ surfaces. ${ }^{15}$ Those results confirm the requirement of a conducting surface with large spin-orbit coupling to arise the giant magnetic moments.

A first remark is related to the constriction for one orientation. The conduction electrons rotate around localized charges and/or localized spins along orbits contained on the surface and consequently one can only have a $z$ component. Therefore, the rotation of an orbital magnetic moment is meaningless since it would mean that the electrons leave the metallic surface. However, according to Eq. (1), for any given $l_{z}$ its reversal does not change the energy provided there is there is a simultaneous reversal of $\left(s_{z}+S_{z}\right)$.

Since for thiol-capped gold films the orbital moment is giant, its contribution is the only one that can be observed in the macroscopic magnetization. Therefore, independently of temperature, the measured anisotropy seems to be infinity given that the orbits are not allowed to rotate, as depicted in Fig. 1(d). When the induced orbital momentum is of a few $\hbar$ units, its associated magnetic moment is of the order of those associated with the spins and both contribute to the measured macroscopic magnetization as is the case for NPs. The localized spins, even though subjected to possible anisotropies independent of the orbital motion, are mainly blocked by the effective spin-orbit field, $H^{*}$, given by Eq. (1).

$$
\mu_{0} H^{*}=\alpha_{r} \hbar^{2} l_{z} / \mu_{B}
$$

This field strength $\mu_{0} H^{*}$ is close to $1000 \mathrm{~T}$ for $\alpha_{r} \hbar^{2}$ $=0.4 \mathrm{eV}$, that is, the case of spins localized on gold surfaces with $l_{z}=1$. Furthermore, for Au films the localized spins are blocked through an effective field $10^{2}$ times higher due to the giant value of $l_{z}$ that can approximate to $10^{2}$. Thus, for the case of gold in both forms films and NPs the enormous strength of $H^{*}\left(10^{5}\right.$ and $10^{3} \mathrm{~T}$, respectively) indicates that $S_{z}$, $s_{z}$, and $l_{z}$ remain stiffly coupled under any applied field. This giant effective field cannot induce any reversal of the magnetic moments since its direction reverses with them, but accounts, however, for the blocking of moments observed in $\mathrm{Au}$ NPs up to room temperature. ${ }^{16}$

The second point deals with the magnetization process. For capped Au films subjected to fields applied along the $z$ axis no hysteresis is observed, as shown in Fig. 1(c). The perpendicular magnetization tends to saturate at relatively low fields compared to the infinity value of the anisotropy constant inferred from the in-plane magnetization curve. This curve shows the reversible character of the magnetization process. The slope of the magnetization curve is a consequence of the demagnetizing field $\left(H_{d}\right)$ that takes a maximum value in the direction perpendicular to the field (for the direction perpendicular to the surface the demagnetizing factor, $N$, is unity, so $\left.H_{d}=-M\right)$. The lack of hysteresis indicates that at zero applied field, the number of orbits with quantum number $l_{z}$ is equal to that with $-l_{z}$ and that transitions between them do not need to overcome any barrier. Consequently, a very weak magnetic field enables $l_{z}$ reversal.

The hysteresis observed for $\mathrm{Au}$ and Pd NPs can be explained as follows. The localized spins are also subjected to the local structural anisotropy with constant $k$ per atom and with an easy axis that we assume is perpendicular to the surface. Therefore, if the localized spin were isolated, they would reverse for an opposite field of strength $H=2 k / \mu_{0} \mu_{B}$. However, the applied field acting on $S$ and trying to reverse it also acts on the orbital moment that is rigidly linked to $S$, through Eq. (1), as illustrated by Fig. 2. Consequently the reversal will take place at a field

$$
H_{K}=\frac{2 k}{\mu_{0} \mu_{B}\left(2 s_{z}+2 S_{Z}+l_{z}\right)} .
$$

When $l_{z}$ is of the order of 100 , as is the case of gold films, the reversal field is negligible, the curve does not present hysteresis, and only demagnetizing effects are observed. For NPs, as $l_{z}$ is of the order of unity, ${ }^{10}$ the reversal is somewhat larger than for thin films (for which $l_{z}$ is larger) so some hysteresis may be still observed. In the case of NPs only those magnetic moments comprised within an $\varphi$ angle around the field axis reverse. (For an applied reversal field $H_{a}$, it is verified $H_{a} \cos \varphi=H_{k}$ ).

It is the more important inference according to Eqs. (1) and (2) that even though the structural anisotropy constant $k$ could be of the order of the usual values for bulk materials, $0.1 \mathrm{meV}$ per atom, the hysteresis does not disappear with temperature in NPs. The strong effective field, $H^{*}$, eliminates the thermal fluctuations effect and the spin proceeds blocked up to temperatures of the order $k_{B} T=0.4 \mathrm{eV}$ or $T=5000 \mathrm{~K}$, independently of the NPs size, provided that $l_{z}$ is different to zero. The magnetic moments are blocked by $H^{*}$, rather than by $k$, and can remain blocked up to above $300 \mathrm{~K}$ when the NPs size is even smaller than $1 \mathrm{~nm}$. Consequently, the magnetization under constant applied field does not depend on the temperature over a broad interval between 5 and $300 \mathrm{~K}$.

In summary, at conducting surfaces, with a strong $\xi^{2} \alpha_{r}$ product, localized electric charge or localized spins can trap electrons in orbits with angular momentum $l_{z}$. This orbital moment cannot rotate but only reverse its value under the action of external magnetic fields. The localized spins are subjected to a strong effective magnetic field due to its coupling to $l_{z}$ via spin-orbit interaction. The reversal of the magnetic moment preserves the rigid coupling between spins and orbital moments. The structural local anisotropy acting on the localized spins gives rise to hysteresis that becomes neg- 
ligible for giant $l_{z}$ values. Thermal motion does not affect the probability jumps over the anisotropy barrier as a consequence of the giant field $H^{*}$ acting on the spin. The intrinsic mechanism underlying the reversal of $l_{z}$ remains to be elucidated. These conclusions allow us to tailor samples at nanoscale with permanent magnetism up to temperatures above room temperature. This new type of magnetism is associated with surfaces where adequate capping and restrictions for orbital momentum rotation enable the induction of giant $H^{*}$. Thus, high spin-orbit surfaces, with localized spins or elec- tric charge distributions and well defined orbits, as has been shown for a few cases, are expected to exhibit amazing magnetic properties completely different to those well known for $3 d$ and $4 f$ elements.

This work has been partially supported by the projects No. NAN2004-09125-C07-05 (Spanish Ministry of Education and Science) and No. 200560F0174 (Spanish Council for Scientific Research).
*Corresponding author. Email address: ahernando@renfe.es

${ }^{1}$ P. Gambardella, S. Rusponi, M. Veronese, S. S. Dhesi, C. Grazioli, A. Dallmeyer, I. Cabria, R. Zeller, P. H. Dederichs, K. Kern, C. Carbone, and H. Brune, Science 300, 1130 (2003).

${ }^{2}$ P. Crespo, R. Litrán, T. C. Rojas, M. Multigner, J. M. de la Fuente, J. C. Sánchez-López, M. A. García, A. Hernando, S. Penadés, and A. Fernández, Phys. Rev. Lett. 93, 087204 (2004).

${ }^{3}$ Y. Yamamoto, T. Miura, M. Suzuki, N. Kawamura, H. Miyagawa, T. Nakamura, K. Kobayashi, T. Teranishi, and H. Hori, Phys. Rev. Lett. 93, 116801 (2004).

${ }^{4}$ B. Sampedro, P. Crespo, A. Hernando, R. Litrán, J. C. Sanchez López, C. López Cartes, A. Fernandez, J. Ramírez, J. González Calbet, and M. Vallet, Phys. Rev. Lett. 91, 237203 (2003).

${ }^{5}$ T. Shinohara, T. Sato, and T. Taniyama, Phys. Rev. Lett. 91, 197201 (2003).

${ }^{6}$ I. Carmeli, G. Leitus, R. Naaman, S. Reich, and Z. Vager, J. Chem. Phys. 18, 10372 (2003).

${ }^{7}$ Z. Vager, I. Carmeli, G. Leitus, S. Reich, and R. Naaman, J. Phys. Chem. Solids 65, 713 (2004).

${ }^{8}$ M. Hegner, P. Wagner, and G. Semenza, Surf. Sci. 291, 39 (1993).

${ }^{9}$ C. Tromas, P. Eaton, J. Mimault, J. Rojo, and S. Penades, Langmuir 21, 6142 (2005)

${ }^{10}$ A. Hernando, P. Crespo, and M. A. Garcia, Phys. Rev. Lett. 96, 057206 (2006).

${ }^{11}$ It is also important to indicate that for the case of localized spins the induction of orbital momentum at the conduction electrons requires the orientation fluctuation frequency, $\nu$, of the localized spin to be smaller than the frequency of the orbital motion, $\nu_{l}$ $=\alpha_{s} S_{z} h$, that for the case of gold becomes $10^{13} \mathrm{~s}^{-1}$. As $\nu$ can be expressed as $\nu_{o} \exp \left(-k^{*} / k_{B} T\right)$ its maximum value is $\nu_{o}$, that is, approximately $10^{10} \mathrm{~s}^{-1}$.

${ }^{12}$ S. LaShell, B. A. McDougall, and E. Jensen, Phys. Rev. Lett. 77, 3419 (1996). See also, Y. M. Koroteev et al., ibid. 93, 046403 (2004).

${ }^{13}$ L. Petersen and P. Hedegard, Surf. Sci. 459, 49 (2000).

${ }^{14}$ E. Barrena, C. Ocal, and M. Salmeron, J. Chem. Phys. 111, 9797 (1999).

${ }^{15}$ In Ref. 6 it is stated that no magnetic moments are found depositing polyalanine molecules onto silicon surfaces. However it is clear from Fig. 1 of this reference that there is a small magnetic component, much smaller than for $\mathrm{Au}$ surfaces, that is easily overcome by the diamagnetism of a $\mathrm{Si}$ substrate. In any case this magnetic moment is not giant.

${ }^{16}$ The results reported in Ref. 1 could be revisited in the framework of these considerations. According to the anisotropy measured per Co atom, $9 \mathrm{meV}$, the effective field $H^{*}$ should be close to $20 \mathrm{~T}$, that allows the localized spins to rotate weakly toward the in-plane direction for applied fields of a few T. The correlation between orbital magnetism and the number of atoms per Co cluster as well as its correlation to the measured anisotropy constant does not suggest that effects as described in this Brief Report have to be discarded. The magnetism of graphite induced by protons irradiation could also be analyzed according to orbital magnetism basis, see for instance, P. Esquinazi et al., Phys. Rev. Lett. 91, 227201 (2003). 